\title{
Second-trimester fetal genetic ultrasonography to detect chromosomal abnormalities
}

\author{
Seong-Yeon Hong* \\ Department of Obstetrics and Gynecology, Catholic University of Daegu, Daegu, Korea
}

Genetic ultrasonography refers to the evaluation of risk of chromosomal abnormalities via various soft sonographic markers. Although the maternal serum test is the primary screening method for chromosomal abnormalities, genetic ultrasonography is also widely used and can help increase detection rates. To date, many soft markers, including choroid plexus cysts, echogenic intracardiac foci, mild ventriculomegaly, nuchal fold thickening, echogenic bowel, mild pyelectasis, short femur and humerus length, and absent or hypoplastic nasal bone, have been reported. An aberrant right subclavian artery was the most novel soft marker introduced. Because these soft markers involve diverse relative risks of chromosomal abnormalities, it is difficult to apply them to clinical practice. To optimize the efficacy of genetic ultrasonography, it is important to understand the precise relative risks of chromosomal abnormalities innumerous soft markers and integrate these risks with each other and the results of maternal serum screening.

Key words: Genetic ultrasonography, Chromosome aberrations, Down syndrome, Soft marker.

\section{Introduction}

The incidence of chromosomal abnormality, specifically trisomies 21,18 , and 13 , is $0.1-0.2 \%$ per live birth. To date, various soft markers have been found to identify chromosomal abnormalities, including trisomy 21 , and new markers are the focus of current research. Unlike fetal structural anomalies and major anomalies, sonographic markers are not pathological per se, and they refer to sonographic findings associated with chromosomal abnormalities. In general, genetic sonogram is the term used to describe sonographic screening for fetal chromosomal abnormalities including trisomy 21 . The most frequently evaluated sonographic markers include choroid plexus cysts (CPCs), echogenic intracardiac foci (EICF), mild ventriculomegaly, nuchal fold thickening (NFT), echogenic bowel, mild pyelectasis, short femur and humerus, and absent or hypoplastic nasal bone. Mild ventriculomegaly is considered a major anomaly, but it can also be regarded as a sonographic marker. The usefulness of the aberrant right subclavian artery (ARSA) as a novel sonographic marker has been reported recently.

As the number of sonographic markers increases, the risk of chromosomal abnormalities also increases. Although the absence of these sonographic markers decreases the risk of chromosomal abnormalities, the relative risks of chromosomal abnormalities reported in numerous studies vary.

Herein, the sonographic markers for evaluating the risk of chromosomal abnormalities, the role of genetic ultrasonography, and an ARSA as a novel soft marker have been described, with a

Received: 12 June 2014, Revised: 10 December 2014, Accepted: 10 December 2014, Published: 31 December 2014

${ }^{*}$ Corresponding author: Seong-Yeon Hong, M.D.

Department of Obstetrics and Gynecology, Catholic University of Daegu, 33 Duryugongwon-ro 17-gil, Nam-gu, Daegu 705-718, Korea.

Tel: +82-53-650-4078, Fax: +82-53-650-4078, E-mail: magu815@cu.ac.kr

Conflict of interest: We declare that we do not have any conflicts of interests.

(c) This is an open-access article distributed under the terms of the Creative Commons Attribution Non-Commercial License (http://creativecommons.org/licenses/by-nc/3.0/) which permits unrestricted non-commercial use, distribution, and reproduction in any medium, provided the original work is properly cited.

(c) Copyright 2014 by the Korean Society of Medical Genetics 
brief review of relatedmeta-analyses.

\section{Sonographic Soft Markers}

\section{Choroid plexus cyst}

A CPC is a small round or ovoid anechoic cyst with a discrete border in the choroid plexus of the lateral ventricle. This can be visualized as a single cyst or multiple cysts and is present unilaterally or bilaterally in lateral ventricles.

The CPC is one of the most controversial sonographic markers with respect to usefulness inthe evaluation of the risk of chromosomal abnormalities, including trisomy 21, particularly in low-risk populations.

CPCs are found in 1-2\% of normal fetuses [1]. The presence of a CPC, particularly when accompanied by other sonographic markers or structural anomalies, is associated with increased risk of chromosomal abnormalities, mainly trisomy 18.

However, when a CPC is an isolated finding without other sonographic markers, its presence does not increase the risk of chromosomal abnormalities including trisomies 21 and 18 [2].

Bronsteen et al. [3] reported the association between risk of trisomy 18 and the presence of an isolated CPC. A total of 49,435 fetuses at 16-25 gestational weeks were included, and CPCs were observed in 1,209 cases (2.3\%), 1,060 of which included an isolated CPC. Fifty of the 49,435 fetuses were diagnosed with trisomy 18 , and CPCs were present in half of those 50 fetuses. However, trisomy 18 was not found in fetuses with isolated CPCs. When fetuses have apparently isolated CPCs without other findings, including hand abnormalities, the risk of trisomy 18 is very low, and invasive chromosomal analysis is not recommended.

The size of the CPC is not associated with the risk of chromosomal abnormalities; furthermore, this risk is not influenced by whether single or multiple CPCs are found or whether they are present unilaterally or bilaterally [4]. Therefore, it is reasonable not to regard a CPC as a soft marker when it is an isolated finding [5].

\section{Echogenic intracardiac foci}

EICF is defined as the presence of focal echogenic dots in the papillary muscle of ventricles, which has the same echogenicity as that of bone. EICF is found in 1.5-4.0\% of pregnancies [6], but the incidence rate reaches 10-30\% in Asian populations [7]. EICF is observed as a single echogenic focus in the majority of cases, but multiple foci are occasionally found. Simpson et al. [8] reported that the most frequent finding was a single echogenic focus in the left ventricle (60\% of cases), with multiple foci observed in 33\% of cases. An echogenic focus in the right ventricle was found in $7 \%$ of cases.

Roberts and Genest [9] first reported the association between EICF and chromosomal abnormalities in 1992, and many subsequent studies have reported that the risk of chromosomal abnormalities increased in the presence of EICF [10-12]. However, because these studies only included high-risk populations, these results cannot be generalized to those with low risk.

In a randomized controlled trial including 12,672 pregnant women, Coco et al. [13] reported that the risk of chromosomal abnormalities increased in the presence of EICF, but lowrisk populations with isolated EICF were not candidates for amniocentesis. In a study including 21,839 women at low risk of chromosomal abnormalities, Filly et al. [1] reported that isolated EICF was found in 626 fetuses (3\%), one of which was diagnosed with Down syndrome. On the basis of these results, they claimed that amniocentesis is not recommended in fetuses with isolated EICF.

\section{Nuchal fold thickening}

$\mathrm{NFT}$ is defined as the soft tissue between the posterior border of an occipital bone and the outer margin of nuchal skin. This can be measured in the transcerebellar plane of the fetal brain, in which the cavum septi pellucidi, frontal horns of the lateral ventricles, thalami, cerebellum, and cisterna magna are all visualized together. If nuchal fold thickness is more than 5 or $6 \mathrm{~mm}$ between gestational weeks 15 and 23, it is considered thickened. When this occurs, it is thought to be one of the strongest sonographic markers indicating risk of chromosomal abnormalities [14].

NFT has reportedly been identified in $80 \%$ of neonates diagnosed with trisomy 21 and is associated with other chromosomal aneuploidies such as trisomies 13 and 18 and monosomyX [15].

What is the optimal threshold of nuchal fold thickness in the estimation of the risk of chromosomal abnormalities? Earlier studies recommended a threshold of $6 \mathrm{~mm}$. However, more recent prospective studies proposed that a threshold of $5 \mathrm{~mm}$ was more valuable, because the nuchal fold is less than $5 \mathrm{~mm}$ thick in most normal fetuses, and much higher sensitivity and a slightly higher false-positive rate have been observed with a threshold of $5 \mathrm{~mm}$ relative to $6 \mathrm{~mm}[16,17]$. 
Bahado-Singh et al. [18] and Locatelli et al. [19] proposed a new method of application for NFF using multiples of medians (MoMs) corresponding to gestational weeks. The concept was based on the finding that nuchal fold thickness was correlated with gestational age in both normal fetuses and those with trisomy 21. Locatelli et al. [19] described nuchal fold thickness according to MoMs as "expected nuchal fold thickness," and Bahado-Singh et al. [18] described it as "gestational age standardized nuchal thickness values".

Benacerraf et al. [20] recommended NFT as the most sensitive sonographic marker in evaluating risk of trisomy 21. Amniocentesis should be recommended in fetuses with isolated NFT, even if they are at low risk of chromosomal abnormalities. Furthermore, fetuses showing NFT are candidates for amniocentesis, although nuchal fold thickness is normalized on follow-up scans.

\section{Echogenic bowel}

Echogenic bowel is a term used as a synonym for hyperechogenic bowel, which suggests that the echogenicity of the small bowel is identical to that of the bone. This finding can be focal, multifocal, or diffuse. Echogenic bowel is identified in 0.2-1.4\% of fetuses during the second trimester [21]. The presence of echogenic bowel is associated with chromosomal anomalies including trisomy 21 and other pathologic conditions such as fetal growth restriction, intestinal bleeding, cystic fibrosis, thalassemia, and toxoplasmosis, other, rubella, cytomegalovirus, and herpes (known as TORCH) infections.

Echogenic bowel can be classified into several grades according to echogenicity of the bowel. Slotnick and Abuhamad [22] classified echogenic bowel into 3 grades according to echogenicity relative to that of iliac bone. Grade 1 included lower echogenicity of the bowel relative to that of the iliac bone; Grade 2 included echogenicity of the bowel identical to that of the iliac bone; and Grade 3 included higher echogenicity of the bowel relative to that of the iliac bone.

With respect to the association between echogenic bowel and trisomy 21, relative risk is reported to be as high as 6.1$6.7[14,23]$. When echogenic bowel is visualized on a secondtrimester scan, a thorough sonographic evaluation should be recommended to identify other sonographic markers or structural anomalies.

\section{Short femur and humerus length}

In general, fetuses with trisomy 21 have short stature with short femurs or humeri. Therefore, numerous studies have examined the association between trisomy 21 and short femur or humerus length. Benacerraf et al. [24] were the first to report this association.

The femur and humerus lengths considered short in the evaluation of risk of chromosomal abnormalities have been studied, with the ratio between the measured and expected femur or humerus length used to identify short femur or humerus length $[24,25]$. Expected femur and humerus length is calculated using the following formulas: expected femur length $=(-9.3105+0.9028 \times$ biparietal diameter $)$ and expected humerus length $=(-7.9404+0.8492 \times$ biparietal diameter $)$. Based on these formulas, femur and humerus lengths are considered short when the ratios of measured to expected lengths are less than 0.91 and 0.89 , respectively.

Bahado-Singh et al. [26] proposed methods using MoMs, which reflected the associations between chromosomal abnormalities and short femur and humerus lengths.

Some researchers have reported that short humerus length is more valuable than short femur length in predicting the risk of trisomy $21[27,28]$. In addition, short humerus length alone is more predictable than short femur and humerus length combined, because the latter reflects the possibility of fetal growth restriction rather than chromosomal abnormalities [23].

\section{Mild pyelectasis}

Mild pyelectasis is defined as an anteroposterior renal pelvis diameter of $4 \mathrm{~mm}$ or greater in the axial plane of the fetus ona second-trimester scan.

Benacerraf et al. [29] were the first to report the association between mild pyelectasis and chromosomal abnormalities including trisomy 21. The association was found in 25\% of fetuses with trisomy 21 and 2.8\% of fetuses with normal chromosomes in women at a high risk of chromosomal abnormalities.

With respect to the association between mild pyelectasis and chromosomal abnormalities, recent studies have not reported a correlation between isolated pyelectasis and chromosomal abnormalities in low-risk populations.

In a large prospective multicenter study including 101,600 births, Chudleigh et al. [30] reported that most pregnancies with isolated mild pyelectasis represented a low risk of chromosomal aneuploidy. Mild pyelectasis was found in 737 fetuses $(0.7 \%)$ in a whole series. Chromosomal abnormalities were found in 12 of these cases (1.7\%), of which 9 involved another sonographic abnormality, one was associated with advanced maternal age, and only two (0.3\%) involved isolated pyelectasis. 
In a study including 25,586 low-risk individuals, Havutcu et al. [31] reported that mild pyelectasis was found in 320 cases (1.25\%), of which 301 involved isolated pyelectasis, and 19 were associated with other soft makers. None of the 315 cases in which delivery occurred in hospitals involved chromosomal abnormalities.

\section{Aberrant right subclavian artery}

The right subclavian artery is a normal branch of the brachiocephalic artery of the aortic arch. An ARSA arises as a variant vessel from the posterior portion of the aortic arch and runs to the right, behind the trachea. Three branches originate from a normal aortic arch: the brachiocephalic artery (which is divided into the right subclavian artery and right common carotid artery), the left common carotid artery, and the left subclavian artery. In contrast to the normal aortic arch, four branches originate from an aortic arch with an ARSA: the right common carotid artery, the left common carotid artery, the left subclavian artery, and the ARSA.

To visualize an ARSA, the color Doppler velocity setting is lowered to $15-30 \mathrm{~cm} / \mathrm{s}$ to facilitate visualization of peripheral vessels [32]. Prior to visualization of an ARSA, the following method is used to find an RSA: 1) find an ascending aorta in 3-vesseltrachea view; 2) tilt the transducer toward the fetal head until the transverse aortic arch is visualized, or visualization of the base of the brachiocephalic artery begins; and 3) tilt the transducer further until an RSA is visualized, or the correct RSA coursing toward the right upper arm can be identified. Normal RSA runs anterior to the trachea and the esophagus, but the ARSA courses behind the trachea and esophagus, toward the right arm.

The ARSA is present in less than 1\% of normal fetuses [33].
However, it is found more frequently in Down syndrome populations [34]. Rathore and Sreenivasan [35] reported that populations with trisomy 21 displayed a high incidence of ARSA at 36\%, but some studies have reported incidences up to 5\% lower than this. The actual incidence is not known.

With respect to the association between the ARSA and other cardiac anomalies, 2.9\% of patients with cardiac anomalies exhibited an ARSA, but the incidence somewhat lower (0.1\%) in patients without cardiac anomalies [34]. Because the ARSA is asymptomatic in the majority of cases, it is found incidentally during autopsy. Yoo et al. [36] reported prenatal diagnosis of the ARSA, but did not describe an association with trisomy 21. Chaoui et al. [32] first reported the ARSA in fetuses with trisomy 21 , which was associated with the ARSA in $35.7 \%$ of cases.

It is true that the ARSA is found more frequently in fetuses with trisomy 21 relative to those without trisomy 21. However, the majority of fetuses with trisomy 21 and the ARSA have additional sonographic markers or other structural anomalies. To date, the estimated risk of trisomy 21 has not been high in fetus with isolated ARSA [37].

\section{Meta-analysis of Second-trimester Markers for Trisomy 21}

Although various sonographic markers have been evaluated for diagnosing Down syndrome, it is difficult to apply sonographic markers to clinical practice because of the diversity in the relative risks reported by researchers. More precise information could be obtained via a meta-analysis of relative risk with respect to such sonographic markers. Agathokleous et

Table 1. Pooled estimated likelihood ratios of soft markers and estimated likelihood ratios of isolated markers [38]

\begin{tabular}{lccc}
\hline \multicolumn{1}{c}{ Marker } & Positive & Negative & Isolated markers \\
\hline EICF & $5.83(5.02-6.77)$ & $0.80(0.75-0.86)$ & 0.95 \\
Ventriculomegaly & $27.52(13.61-55.68)$ & $0.94(0.91-0.98)$ & 3.81 \\
Nuchal fold thickening & $23.30(14.35-37.83)$ & $0.80(0.74-0.85)$ & 3.79 \\
Echogenic bowel & $11.44(9.05-14.47)$ & $0.90(0.86-0.94)$ & 1.65 \\
Mild pyelectasis & $7.63(6.11-9.51)$ & $0.92(0.89-0.96)$ & 1.08 \\
Short humerus & $4.81(3.49-6.62)$ & $0.74(0.63-0.88)$ & 0.78 \\
Short femur & $3.72(2.79-4.97)$ & $0.80(0.73-0.88)$ & 0.61 \\
ARSA & $21.48(11.48-40.19)$ & $0.71(0.57-0.88)$ & 3.94 \\
Absent or hypoplastic nasal bone & $23.27(14.23-38.06)$ & $0.46(0.36-0.58)$ & 6.58 \\
\hline
\end{tabular}

Values are presented as likelihood ratio (95\% confidence interval).

EICF: echogenic intracardiac foci; ARSA: aberrant right subclavian artery.

Adapted from the article of Agathokleous et al. (Ultrasound Obstet Gynecol 2013;41:247-61). 
al. [38] conducted a meta-analysis involving this issue in 2013, which included a total of 48 studies. The soft markers evaluated in their study were EICF, ventriculomegaly, NFT, echogenic bowel, mild pyelectasis, short femur, short humerus, ARSA, and absent or hypoplastic nasal bone. Pooled estimated positive and negative likelihood ratios for each soft marker are shown in Table 1.

If none of the soft markers, including short femur rather than short humerus, were observed, the combined negative likelihood ratio was 0.13 (95\% confidence interval [CI], 0.050.29), and such findings reduced pretest relative risk of Down syndrome based on maternal age and maternal serum screening 7.7 times. Whereas, if none of the soft markers, including short humerusrather than short femur, were observed, combined negative likelihood ratio was $0.12(95 \% \mathrm{Cl}, 0.06-0.29)$, and this reduced pretest relative risk 8.3 times. The effect of isolated markers alone on pretest risk of trisomy 21 was negligible. However, ventriculomegaly, NFT, or ARSA increased relative risk 3-4 times, and hypoplastic nasal bone increased it 6-7 times. If the ARSA and nasal bone were not included, the negative likelihood ratio for Down syndrome was 0.40, which resulted in pretest relative risk being reduced 2.5 times.

\section{Clinical Utilization of Genetic Ultrasonography}

Although it is clear that the presence of sonographic markers increases the risk of chromosomal abnormalities, application and integration of these markers to clinical practice is difficult and can be problematic. Various sonographic markers can be used to assist the detection of chromosomal abnormalities with increased sensitivity; however, they result in increased falsepositive rates. Therefore, methods that maximize sensitivity in high-risk populations and minimize false-positive rates in lowrisk populations are required.

To date, there have been many approaches to the utilization of genetic ultrasonography. Two of the most acceptable approaches are the Index Scoring System (ISS) and Age-adjusted Ultrasound Risk Assessment (AAURA).

\section{Index Scoring System}

Benacerraf et al. [39] proposed a sonographic scoring index in 1992. Using this system, each sonographic marker was converted to form a numerical point value, based on sensitivity, with which to detect chromosomal abnormalities. A total
Table 2. Index Scoring System [40]

\begin{tabular}{lc}
\multicolumn{1}{c}{ Variable } & Score \\
\hline Finding & \\
\hline Major anomaly & 2 \\
\hline Thickened nuchal fold & 2 \\
\hline Short femur & 1 \\
\hline Short humerus & 1 \\
\hline Pyelectasis & 1 \\
\hline Hyperechoic bowel & 1 \\
\hline Echogenic intracardiac focus & 1 \\
With additional points for maternal age (yr) & 0 \\
\hline$<35$ & 1 \\
$\geq 35$ and $<40$ & 2 \\
\hline 40 & \\
\hline
\end{tabular}

Adapted from the article of Bromley et al. (Ultrasound Obstet Gynecol 1997;10:321-4).

score of 2 or greater was regarded as the threshold at which to perform an amniocentesis. This method represented 75.5\% sensitivity with a $5.7 \%$ false-positive rate.

In 1997, Bromley et al. [40] modified and revised this system through the incorporation of maternal age factors into the previous sonographic scoring index, which was proposed by Benacerraf et al. [39] (Table 2). This modified method was applied to both high- and low-risk populations and yielded increased sensitivity (86.8\%) but also increased the false-positive rate (27.1\%).

\section{Age-adjusted Ultrasound Risk Assessment}

Nyberg et al. [41] reported an AAURA in 1998. Prior risk, based on maternal age and serum screening, was adjusted by multiplying the likelihood ratios of sonographic markers. This method represents $61.5 \%$ sensitivity with a $4 \%$ false-positive rate in women younger than 35 years. In women aged 3539 years with normal ultrasonographic findings, this method reduced the rate of amniocentesis from 100\% to 12.5\% but missed $1 / 3$ of the fetuses with trisomy 21 . Subsequent to the proposal of AAURA by Nyberg et al., a number of studies were conducted using different likelihood ratios for sonographic markers.

\section{Comparison between the ISS and AAURA}

Winter et al. [42] reported a comparison between the ISS and AAURA. Genetic amniocentesis was performed in 3,303 high-risk women, and all patients were evaluated with both sonographic methods. They concluded that the two methods were equally effective in screening for trisomy 21 , with a 
detection rate of approximately $50 \%$ and a $5 \%$ false-positive rate.

\section{Conclusion}

To date, many methods of screening for chromosomal abnormalities, including trisomy 21 , have been evaluated. A method that uses maternal age and serum screening is the simplest and most widely used. However, sonographic screening methods are used adjunctively. Although it is impossible to diagnose chromosomal abnormalities prenatally usingsonographic methods alone, the value of genetic ultrasonography is increasing as it benefits from technical and scientific advances in ultrasound diagnostic equipment.

Caution should be exercised in applying genetic ultrasonography to clinical practice, with respect to the following issues. Because the detection rates for maternal serum screening tests such as the triple marker test or quadruple test were low in the past (60-75\%), genetic ultrasonography strongly influences decisions concerning invasive chromosomal analysis such as amniocentesis. As the detection rates for combined first- and second-trimester maternal serum screening tests such as the integrated test or sequential test have improved substantially during the past 10 years, modification of previous risk via genetic ultrasonography is a matter of debate.

It is impossible to diagnose chromosomal abnormalities with either maternal serum screening or genetic ultrasonography. Integration of the two methods, rather than a mere combination, is preferable.

Efforts to discover new soft markers should be continued, and meta-analyses that evaluate previous soft markers are also required. Furthermore, trials conducted to establish standard guidelines are also needed to facilitate the application of soft markers to clinical practice.

\section{References}

1. Filly RA, Benacerraf BR, Nyberg DA, Hobbins JC. Choroid plexus cyst and echogenic intracardiac focus in women at low risk for chromosomal anomalies. J Ultrasound Med 2004;23:447-9.

2. Yoder PR, Sabbagha RE, Gross SJ, Zelop CM. The second-trimester fetus with isolated choroid plexus cysts: a meta-analysis of risk of trisomies 18 and 21. Obstet Gynecol 1999;93:869-72.

3. Bronsteen $\mathrm{R}$, Lee $\mathbf{W}$, Vettraino IM, Huang $\mathrm{R}$, Comstock $\mathrm{CH}$. Secondtrimester sonography and trisomy 18: the significance of isolated choroid plexus cysts after an examination that includes the fetal hands. J Ultrasound Med 2004;23:241-5.

4. Gupta JK, Cave M, Lilford RJ, Farrell TA, Irving HC, Mason G, et al. Clinical significance of fetal choroid plexus cysts. Lancet 1995;346:724-9.

5. Bahado-Singh RO, Oz UA, Mendilcioglu I, Mahoney MJ. The midtrimester genetic sonogram. Semin Perinatol 2005;29:209-14.

6. Sotiriadis A, Makrydimas G, loannidis JP. Diagnostic performance of intracardiac echogenic foci for Down syndrome: a meta-analysis. Obstet Gynecol 2003;101:1009-16.

7. Shipp TD, Bromley B, Lieberman E, Benacerraf BR. The frequency of the detection of fetal echogenic intracardiac foci with respect to maternal race. Ultrasound Obstet Gynecol 2000;15:460-2.

8. Simpson JM, Cook A, Sharland G. The significance of echogenic foci in the fetal heart: a prospective study of 228 cases. Ultrasound Obstet Gynecol 1996;8:225-8.

9. Roberts DJ, Genest D. Cardiac histologic pathology characteristic of trisomies 13 and 21. Hum Pathol 1992;23:1130-40.

10. Bromley B, Lieberman E, Laboda L, Benacerraf BR. Echogenic intracardiac focus: a sonographic sign for fetal Down syndrome. Obstet Gynecol 1995;86:998-1001.

11. Manning JE, Ragavendra N, Sayre J, Laifer-Narin SL, Melany ML, Grant EG, et al. Significance of fetal intracardiac echogenic foci in relation to trisomy 21: a prospective sonographic study of high-risk pregnant women. AJR Am J Roentgenol 1998;170:1083-4.

12. Vibhakar NI, Budorick NE, Scioscia AL, Harby LD, Mullen ML, Sklansky MS. Prevalence of aneuploidy with a cardiac intraventricular echogenic focus in an at-risk patient population. J Ultrasound Med 1999;18:265-8; quiz 269-70.

13. Coco $C$, Jeanty $P$, Jeanty $C$. An isolated echogenic heart focus is not an indication for amniocentesis in 12,672 unselected patients. J Ultrasound Med 2004;23:489-96.

14. Nyberg DA, Souter VL, El-Bastawissi A, Young S, Luthhardt F, Luthy DA. Isolated sonographic markers for detection of fetal Down syndrome in the second trimester of pregnancy. J Ultrasound Med 2001;20:1053-63.

15. Hall B. Mongolism in newborn infants. An examination of the criteria for recognition and some speculations on the pathogenic activity of the chromosomal abnormality. Clin Pediatr (Phila) 1966;5:4-12.

16. Borrell A, Costa D, Martinez JM, Delgado RD, Casals E, Ojuel J, et al. Early midtrimester fetal nuchal thickness: effectiveness as a marker of Down syndrome. Am J Obstet Gynecol 1996;175:45-9.

17. Gray DL, Crane JP. Optimal nuchal skin-fold thresholds based on 
gestational age for prenatal detection of Down syndrome. Am J Obstet Gynecol 1994;171:1282-6.

18. Bahado-Singh RO, Oz UA, Kovanci E, Deren O, Feather M, Hsu CD, et al. Gestational age standardized nuchal thickness values for estimating mid-trimester Down's syndrome risk. J Matern Fetal Med 1999;8:37-43.

19. Locatelli A, Piccoli MG, Vergani P, Mariani E, Ghidini A, Mariani S, et al. Critical appraisal of the use of nuchal fold thickness measurements for the prediction of Down syndrome. Am J Obstet Gynecol 2000;182:192-7.

20. Benacerraf BR, Laboda LA, Frigoletto FD. Thickened nuchal fold in fetuses not at risk for aneuploidy. Radiology 1992;184:239-42.

21. Rochon M, Eddleman K. Controversial ultrasound findings. Obstet Gynecol Clin North Am 2004;31:61-99.

22. Slotnick RN, Abuhamad AZ. Prognostic implications of fetal echogenic bowel. Lancet 1996;347:85-7.

23. Smith-Bindman R, Hosmer W, Feldstein VA, Deeks JJ, Goldberg JD. Second-trimester ultrasound to detect fetuses with Down syndrome: a meta-analysis. JAMA 2001;285:1044-55.

24. Benacerraf $B R$, Neuberg D, Frigoletto FD Jr. Humeral shortening in second-trimester fetuses with Down syndrome. Obstet Gynecol 1991;77:223-7.

25. Benacerraf BR, Cnann A, Gelman R, Laboda LA, Frigoletto FD Jr. Can sonographers reliably identify anatomic features associated with Down syndrome in fetuses? Radiology 1989;173:377-80.

26. Bahado-Singh RO, Oz AU, Kovanci E, Deren O, Copel J, Baumgarten $A$, et al. New Down syndrome screening algorithm: ultrasonographic biometry and multiple serum markers combined with maternal age. Am J Obstet Gynecol 1998;179:1627-31.

27. Johnson MP, Michaelson JE, Barr M Jr, Treadwell MC, Hume RF Jr, Dombrowski MP, et al. Combining humerus and femur length for improved ultrasonographic identification of pregnancies at increased risk for trisomy 21. Am J Obstet Gynecol 1995;172:1229-35.

28. Nyberg DA, Resta RG, Luthy DA, Hickok DE, Williams MA. Humerus and femur length shortening in the detection of Down's syndrome. Am J Obstet Gynecol 1993;168:534-8.

29. Benacerraf BR, Mandell J, Estroff JA, Harlow BL, Frigoletto FD Jr. Fetal pyelectasis: a possible association with Down syndrome. Obstet Gynecol 1990;76:58-60.

30. Chudleigh PM, Chitty LS, Pembrey M, Campbell S. The association of aneuploidy and mild fetal pyelectasis in an unselected population: the results of a multicenter study. Ultrasound Obstet Gynecol 2001;17:197-202.

31. Havutcu AE, Nikolopoulos G, Adinkra P, Lamont RF. The association between fetal pyelectasis on second trimester ultrasound scan and aneuploidy among 25,586 low risk unselected women. Prenat Diagn 2002;22:1201-6.

32. Chaoui R, Heling KS, Sarioglu N, Schwabe M, Dankof A, Bollmann R. Aberrant right subclavian artery as a new cardiac sign in second- and third-trimester fetuses with Down syndrome. Am J Obstet Gynecol 2005;192:257-63.

33. Edwards JE. Malformations of the aortic arch system manifested as vascular rings. Lab Invest 1953;2:56-75.

34. Zapata $H_{1}$ Edwards JE, Titus JL. Aberrant right subclavian artery with left aortic arch: associated cardiac anomalies. Pediatr Cardiol 1993;14:159-61.

35. Rathore $\mathrm{MH}$, Sreenivasan W. Vertebral and right subclavian artery abnormalities in the Down syndrome. Am J Cardiol 1989;63:1528-9.

36. Yoo SJ, Min JY, Lee YH, Roman K, Jaeggi E, Smallhorn J. Fetal sonographic diagnosis of aortic arch anomalies. Ultrasound Obstet Gynecol 2003;22:535-46.

37. De León-Luis J, Gámez F, Bravo C, Tenías JM, Arias Á, Pérez $R$, et al. Second-trimester fetal aberrant right subclavian artery: original study, systematic review and meta-analysis of performance in detection of Down syndrome. Ultrasound Obstet Gynecol 2014;44:147-53.

38. Agathokleous M, Chaveeva P, Poon LC, Kosinski P, Nicolaides $\mathrm{KH}$. Meta-analysis of second-trimester markers for trisomy 21. Ultrasound Obstet Gynecol 2013;41:247-61.

39. Benacerraf BR, Neuberg D, Bromley B, Frigoletto FD Jr. Sonographic scoring index for prenatal detection of chromosomal abnormalities. J Ultrasound Med 1992;11:449-58.

40. Bromley $B$, Lieberman $E$, Benacerraf BR. The incorporation of maternal age into the sonographic scoring index for the detection at 14-20 weeks of fetuses with Down's syndrome. Ultrasound Obstet Gynecol 1997;10:321-4.

41. Nyberg DA, Luthy DA, Resta RG, Nyberg BC, Williams MA. Ageadjusted ultrasound risk assessment for fetal Down's syndrome during the second trimester: description of the method and analysis of 142 cases. Ultrasound Obstet Gynecol 1998;12:8-14.

42. Winter TC, Uhrich SB, Souter VL, Nyberg DA. The "genetic sonogram": comparison of the index scoring system with the age-adjusted US risk assessment. Radiology 2000;215:775-82. 